\title{
Review: psychotropic drugs increase the risk of falls in older people
}

\author{
Leipzig RM, Cumming RG, Tinetti ME. Drugs and falls in older people: a systematic review and meta-analysis: I. Psychotropic \\ drugs. J Am Geriatr Soc 1999 Jan;47:30-9.
}

\section{Question}

What is the association between psychotropic drugs and falls in older people?

\section{Data sources}

Published studies in the English language were identified by using Medline (1966 to March 1996) with the search terms accidents, accidental falls, aged, or age factors; reviewing bibliographies of papers; and contacting experts.

\section{Study selection}

Studies were selected if they assessed the association between psychotropic drug use, including antidepressants, neuroleptics, sedatives or hypnotics, or long or short acting benzodiazepines, and falls in people $\geqslant 60$ years of age.

\section{Data extraction}

Data were extracted on study and patient characteristics; fall assessment and definition; drugs; number of falls associated with benzodiazepines, antidepressants, neuroleptics, hypnotics, or sedatives; and the temporal relation between drug assessment, fall assessment, and the index fall.

\section{Main results}

1043 studies were identified and 40 met the inclusion criteria. $70 \%$ of studies defined fall as a non-syncopal event that was not attributable to sustaining a violent blow, loss of consciousness, stroke, or epileptic seizure. 7 studies of community living older people had a $36 \%$ annual incidence of falls and a 34\% incidence of psychotropic drug use, and 2 studies of people in long term care facilities had a 58\% 6-12 month incidence of falls and a
$69 \%$ incidence of psychotropic drug use. 37 studies had follow up $\geqslant 6$ months. Using fixed effects meta-analysis techniques, individual and combinations of psychotropic drugs increased the risk of $\geqslant 1$ fall (table). Neuroleptic drugs increased the risk of falls in community living people (odds ratio [OR] 1.7, 95\% CI 1.4 to 2.0) but decreased the risk in people in hospital (OR 0.4, CI 0.2 to 0.8 ). The risk of falls was not different between short and long acting benzodiazepines or between people with $\geqslant 1$ fall and those with $\geqslant 2$ falls.

\section{Conclusion}

Psychotropic drugs are associated with a small increase in risk of falls in older people.

Weighted odds ratio $(O R)$ for the association between various psychotropic drug classes v placebo and $\geqslant 1$ fall in older people

\begin{tabular}{llll}
\hline Drug class & $\begin{array}{l}\text { Number of } \\
\text { studies }\end{array}$ & $\begin{array}{l}\text { Number of } \\
\text { cohort studies }\end{array}$ & Weighted OR (95\% CI) \\
\hline Any psychotropic drug & 19 & 11 & $1.7(1.5$ to 2.0$)$ \\
Antidepressants & 27 & 11 & $1.7(1.4$ to 2.0$)$ \\
Neuroleptics & 22 & 10 & $1.5(1.3$ to 1.8$)$ \\
TCAs & 12 & 6 & $1.5(1.1$ to 2.0$)$ \\
Sedative/hypnotics & 22 & 9 & $1.5(1.4$ to 1.7$)$ \\
Benzodiazepines & 13 & 8 & $1.5(1.2$ to 1.8$)$ \\
\hline
\end{tabular}

TCAs $=$ tricyclic antidepressants

Source of funding: not stated.

For correspondence:Dr R M Leipzig, Department of Geriatrics and Adult Development, Mount Sinai School of Medicine, 1 Gustave Levy Place, Box 1070, New York, NY 10029-6574, USA. Fax +1 212 8609737.

Abstract and commentary also published in ACP Journal Club.

\section{Commentary}

First described 25 years ago, ${ }^{1}$ the relation between psychotropic drug use and falls has been widely studied. Leipzig et al systematically reviewed 40 studies and found that older people who use psychotropic drugs, compared with those who do not, have about a $50 \%$ increased incidence of falls.

It is biologically plausible that these agents could cause falls. Most psychotropic drugs produce sedation and increased postural sway in older people. ${ }^{2}$ Also, there is remarkable consistency across studies that older people who use these drugs are more likely to fall than those who do not. The relative risk of falls associated with psychotropic drug use usually approaches 1.5 to 2.0. A dose response relation has been shown in several studies; people who use higher doses or multiple psychotropic drugs have the highest risk of falls, as shown in this systematic review.
2 lines of evidence make this association more difficult to define. Firstly, there is a lack of specificity in the association. Despite wide variation in side effects across the spectrum of psychotropic drugs, the risk of falls is similar across all classes of agents. Secondly, none of the observational studies have provided adequate control for "confounding by indication." People who are prescribed the drugs are more likely to fall because the symptoms treated by these agents (eg, anxiety, depression, and agitation) place them at a higher risk of falls. Studies in other areas have used a propensity score to quantify this type of confounding.

Given these uncertainties, psychotropic drugs should not be withheld in settings where they have shown effectiveness solely because they may increase the risk of falls. Older adults with depression should be treated with antidepressants, and I prefer nortriptyline as a first line agent. Older adults with chronic psychosis should be managed with antipsychotic drugs, and I prefer haloperidol as a first line agent. The decision to use anxiolytics, hypnotics, and antipsychotics to manage symptoms of agitation, anxiety, and poor sleep in older adults must be evaluated case by case and those who use these drugs should be cautioned about the risk of falls.

Ronald I Shorr, MD, MS Methodist Healthcare Memphis, Tennessee, USA

1 Ballinger BR, Ramsay AC. Accidents and drug treatment in a psychiatric hospital. $\mathrm{Br} J$ Psychiatry 1975;126:462-3.

2 Robin DW, Hasan SS, Edeki T, et al. Increased baseline sway contributes to increased losses of balance in older people following triazolam. JAm Geriatr Soc 1996;44:300-4.

3 Rubin DB. Estimating causal effects from large data sets using propensity scores. Ann Intern Med 1997;127:757-63. 